\title{
DETERMINAÇÃO DE ACETOÍNA E METANOL EM VINAGRES DE VINHO BRASILEIROS
}

\author{
LUIZANTENOR RIZZON * \\ ALBERTO MIELE **
}

\begin{abstract}
Visou-se a caracterização do vinagre de vinho brasileiro, mediante determinação do teor de acetoína e de metanol. Analisaram-se 29 amostras de diferentes marcas comerciais de vinagre de vinho tinto e 18 de vinagre de vinho branco. As análises de acetoína e de metanol foram efetuadas mediante cromatografia a gás. Constatou-se variabilidade acentuada no teor de acetoína e de metanol do vinagre de vinho brasileiro. Detectou-se que $61,0 \%$ dos vinagres de vinho branco e $39,4 \%$ dos vinagres de vinho tinto apresentaram menos de $40 \mathrm{mg} / \mathrm{L}$ de acetoína. Observou-se, também, que $66,6 \%$ dos vinagres de vinho branco apresentaram menos de $25 \mathrm{mg} / \mathrm{L}$ de metanol e $53,6 \%$ dos vinagres de vinho tinto menos de $50 \mathrm{mg} / \mathrm{L}$. O estabelecimento de limite mínimo pela legislação brasileira para a acetoína e o metanol do vinagre de vinho contribuiria para a determinação da genuinidade desse produto. A cromatografia a gás mostrou-se adequada para a determinação da acetoína e do metanol nos vinagres de vinho.
\end{abstract}

PALAVRAS-CHAVE: ENOLOGIA; VINAGRE DE VINHO; COMPOSTOS VOLÁTEIS; ACETOÍNA; METANOL.

\section{INTRODUÇÃO}

Vinagre é o produto obtido, exclusivamente, da fermentação acética do vinho. Outros produtos denominados fermentados acéticos são elaborados a partir de mostos açucarados que, inicialmente, foram submetidos à fermentação alcoólica e, posteriormente, ao processo de acetificação.

A grande disponibilidade de cana-de-açúcar no Brasil e a diferença acentuada de preço em relação à uva promoveram a concorrência de produtos elaborados a partir do álcool de cana-de-açúcar com os vinagres

* Engenheiro-Agrônomo, Pesquisador, Embrapa Uva e Vinho, Bento Gonçalves, RS (e-mail: rizzon@cnpuv.embrapa.br).

** Engenheiro-Agrônomo, Pesquisador, Embrapa Uva e Vinho. Bolsista do CNPq (email: miele@cnpuv.embrapa.br). 
de vinho. Para garantir a genuinidade do vinagre de vinho brasileiro foi oficializado método analítico por espectrometria de massa, que permite determinar a origem do ácido acético do vinagre pelo estudo da proporção dos isótopos estáveis ${ }^{13} \mathrm{C} /{ }^{12} \mathrm{C}$ (BRASIL, 2000). O vinagre de vinho genuíno deve conter ácido tartárico, compostos fenólicos (especialmente quando elaborados com vinho tinto), relação potássio/sódio elevada, teor de prolina, metanol e de polilcoóis proporcionais aos encontrados no vinho, além de acetoína. A determinação de acetoína e de metanol, mediante cromatografia a gás, pode contribuir para a caracterização dos vinagres de vinho brasileiros. A acetoína é um dos componentes qualitativamente mais importantes e sempre presente no vinagre de vinho (MARCHENA, 1972; MECCA et al., 1979; TRONCOSO e GUSMÁN, 1987; LLAGUNO e POLO, 1991).

A acetoína forma-se em pequena quantidade no decorrer do processo fermentativo, mediante síntese do $\alpha$-acetolactato pela ação das leveduras, da condensação de duas moléculas de aldeído, ou uma de acetaldeído e outra de acetil coenzima (BAUMES, 1998). A acetoína também é formada a partir do ácido cítrico, via ácido pirúvico. No entanto, tanto as leveduras como as bactérias láticas podem reduzir o diacetil em acetoína e em 2,3butanodiol (SALMON, 1998). No vinho, a acetoína está presente em teores médios variáveis de $15 \mathrm{mg} / \mathrm{L}$ a $23 \mathrm{mg} / \mathrm{L}$, mas vinhos alterados podem conter até $140 \mathrm{mg} / \mathrm{L}$ (SHINOHARA et al., 1979; ZMIROU-BONNAMOUR, 1984; RIZZON et al., 1997).

$\mathrm{Na}$ acetificação, a quantidade de acetoína é formada pela via do ácido pirúvico e, principalmente, pela oxidação do 2,3-butanodiol presente em quantidade elevada no vinho. Por essa razão o teor de acetoína do vinagre é mais elevado em relação ao do vinho. Portanto, a quantidade de acetoína no vinagre depende, principalmente, do teor de substâncias precursoras de sua síntese existentes no vinho. O processo rápido de acetificação com bactéria acética submersa forma menos acetoína em relação aos processos lentos. MECCA et al. (1979) encontraram teores médios de acetoína em vinagres italianos variáveis de $60 \mathrm{mg} / \mathrm{L}$ a $150 \mathrm{mg} / \mathrm{L}$, podendo alcançar $800 \mathrm{mg} / \mathrm{L}$ nos produtos alterados. TRONCOSO e GUSMÁN (1987) detectaram teor de acetoína acima de $200 \mathrm{mg} / \mathrm{L}$ em vinagres espanhóis. GALOPPINI e ROTINI (1956) consideram que o vinagre de vinho deve apresentar mais de $400 \mathrm{mg} / \mathrm{L}$ de acetoína e CURZEL (1955) estabeleceu em $200 \mathrm{mg} / \mathrm{L}$ o teor mínimo de acetoína para o vinagre de vinho. A legislação italiana não determina teor mínimo de acetoína para o vinagre de vinho (MECCA et al., 1979) e a legislação espanhola limita em 40 mg/L o mínimo para acetoína em vinagres de vinho (LLAGUNO e POLO, 1991). Embora a legislação brasileira não estabeleça limite para o teor de 
acetoína (BRASIL, 1974) do vinagre de vinho brasileiro TAKEMOTO (2000) detectou teor variável de $4,7 \mathrm{mg} / \mathrm{L}$ a $437,4 \mathrm{mg} / \mathrm{L}$ em vinagres comerciais.

O metanol é um álcool encontrado normalmente em pequena quantidade nos sucos de frutas (FENOCCHIO e MENDES, 1972; LEE at al., 1979), no vinho (PISANTI, 1961; FENOCCHIO e MENDES, 1973; BERTRAND, 1975; LEE et al., 1975; GNEKOW e OUGH, 1976; RIZZON, 1985) e no vinagre (MECCA et al., 1979; CORDONNIER, 1987; LLAGUNO e POLO, 1991; RIZZON e MIELE, 1998).

No vinho, o metanol é formado a partir da hidrólise da pectina da uva e liberado por ocasião da maceração no processo de vinificação. A quantidade encontrada no vinho depende do sistema de vinificação, do cultivar da videira e do tempo de maceração (RIZZON, 1985; RIZZON et al., 1999). No vinagre, o metanol provém do vinho utilizado para acetificação e como não é oxidado pelas bactérias acéticas, a quantidade inicialmente presente no vinho passa para o vinagre. O teor de metanol no vinagre de vinho branco italiano varia entre $20 \mathrm{mg} / \mathrm{L}$ a $30 \mathrm{mg} / \mathrm{L}$, enquanto que no vinagre de vinho tinto varia de $30 \mathrm{mg} / \mathrm{L}$ a $90 \mathrm{mg} / \mathrm{L}$ (MECCA et al., 1979). O teor máximo de metanol para vinagre espanhol é de $1 \mathrm{~g} / \mathrm{L}$ (LLAGUNO e POLO, 1991). A legislação brasileira não estabelece limites para o teor de metanol dos vinagres (BRASIL, 1974).

O objetivo do presente trabalho foi caracterizar o vinagre de vinho brasileiro, mediante determinação do teor de acetoína e de metanol.

\section{MATERIAL E MÉTODOS}

Foram analisados 29 vinagres de vinho tinto e 18 vinagres de vinho branco, representando as marcas comerciais disponíveis em Bento Gonçalves RS, na ocasião. As amostras foram coletadas por técnicos do Serviço de Inspeção Federal do Ministério da Agricultura junto a supermercados e encaminhadas para análise na Embrapa Uva e Vinho.

Utilizou-se para a determinação da acetoína cromatógrafo a gás, marca CG modelo 3537D, equipado com detector de ionização de chama e coluna empacotada de aço inoxidável de 6,0 m de comprimento e 1/8" de diâmetro interno, com $10 \%$ de Carbowax sobre Chromosorb (SHINOHARA et al., 1979). A temperatura da coluna foi de $130^{\circ} \mathrm{C}$, em isoterma, a do vaporizador de $193^{\circ} \mathrm{C}$ e a do detector de $215^{\circ} \mathrm{C}$. O gás de arraste foi o nitrogênio, na vazão de $30 \mathrm{~mL} / \mathrm{min}$. 
A acidez do vinagre foi neutralizada com solução a $10 \%$ de óxido de cálcio p.a. e o vinagre neutralizado foi filtrado em filtro de papel. Separouse alíquota de $20 \mathrm{~mL}$, sendo adicionados $2 \mathrm{~mL}$ de solução de 2-octanol (400 mg/L) (padrão interno). Homogeneizou-se a amostra por $5 \mathrm{~min}$, com agitador magnético, em frasco fechado. As determinações foram feitas em triplicata, sendo utilizado o valor médio obtido. Da amostra de vinagre, assim preparada, foram injetados $3 \mu \mathrm{L}$ no cromatógrafo. Utilizou-se o 2octanol como padrão interno devido ao seu tempo de retenção nas condições de análise e para reduzir o erro analítico. Foram empregados solventes de qualidade analítica para cromatografia a gás.

Para a análise do metanol utilizou-se cromatógrafo a gás, marca CG 3537S, equipado com detector de ionização de chama e coluna de vidro empacotada de 5,0 m de comprimento e 1/8" de diâmetro interno, com $5 \%$ de Carbowax 400 mais $2 \%$ de Hallcomid M18 OL sobre Chromosorb WHP. A temperatura da coluna foi de $80^{\circ} \mathrm{C}$, em isoterma, a do vaporizador de $170^{\circ} \mathrm{C}$ e a do detector de $185^{\circ} \mathrm{C}$. O gás de arraste foi o nitrogênio, na vazão de $30 \mathrm{~mL} / \mathrm{min}$ (BERTRAND, 1975).

\section{RESULTADOS E DISCUSSÃO}

O teor de acetoína e de metanol dos vinagres de vinho brasileiros são indicados na Tabela 1.

\section{TABELA 1 - TEOR DE ACETOÍNA E DE METANOL EM VINAGRES DE VINHO TINTO E DE VINHO BRANCO BRASILEIROS}

\begin{tabular}{|c|c|c|c|c|}
\hline \multirow[b]{2}{*}{ Concentra $\stackrel{a}{0}$} & \multicolumn{2}{|c|}{ Aceto na (mg/L) } & \multicolumn{2}{|c|}{ Metanol (mg/L) } \\
\hline & $\begin{array}{l}\text { Vinagre de } \\
\text { vinho tinto }\end{array}$ & $\begin{array}{l}\text { Vinagre de } \\
\text { vinho branco }\end{array}$ & $\begin{array}{l}\text { Vinagre de } \\
\text { vinho tinto }\end{array}$ & $\begin{array}{l}\text { Vinagre de } \\
\text { vinho branco }\end{array}$ \\
\hline M nimo & 9,0 & 12,4 & 3,0 & 0,1 \\
\hline MAximo & $1.182,0$ & $1.006,0$ & 117,0 & 104,5 \\
\hline Mødio* & $284,1 \mathrm{a}$ & $163,7 \mathrm{a}$ & 49,0 a & $23,0 \mathrm{~b}$ \\
\hline $\begin{array}{l}\text { Intervalo de } \\
\text { confian } a^{\star *}\end{array}$ & $94,7 \quad 473,4$ & $16,6 \quad 344,3$ & $30,4 \quad 67,6$ & $0,2 \quad 46,2$ \\
\hline CV (\%) & 130,1 & 161,3 & 72,4 & 147,4 \\
\hline
\end{tabular}

* Médias seguidas por letras distintas na linha diferem entre si ao nível de $5 \%$ de probabilidade. Prob. $>\mathrm{F}=0,23387$ para a acetoína e Prob. $>\mathrm{F}=0,01670$ para 0 metanol.

** Nível de probabilidade do intervalo de confiança, 99\%.

$\mathrm{CV}=$ Coeficiente de variação. 
Constatou-se variabilidade acentuada tanto na concentração de acetoína como na de metanol nos vinagres analisados. Os valores mínimos detectados de acetoína nos vinagres de vinho branco e nos de vinho tinto foram considerados baixos em relação aos vinagres de vinho de outros países. Os teores máximos de acetoína, além de caracterizar a genuinidade, são indicativos de vinagres elaborados pelo processo lento que sintetizam maior quantidade desse composto.

Não foi observada diferença significativa entre o teor de acetoína do vinagre de vinho branco e o do vinagre de vinho tinto. O vinagre de vinho tinto geralmente apresenta teor de acetoína mais elevado que o vinagre de vinho branco devido à participação, de modo generalizado, da fermentação malolática e do teor mais elevado de 2,3-butanodiol no vinho tinto. Essa diferença, no entanto, não se refletiu no vinagre.

O intervalo de confiança detectado para os vinagres analisados foi muito amplo, devido predominância na utilização do sistema "Frings" para acetificação. Além disso, o vinho comum, especialmente o tinto, matériaprima mais disponível para elaboração do vinagre, apresenta uniformidade na sua composição.

A distribuição percentual das amostras de vinagre analisadas em diferentes níveis de acetoína está indicada na Figura 1.

\section{FIGURA 1 - DISTRIBUIÇÃO DO TEOR DE ACETOÍNA EM VINAGRES DE VINHO TINTO E DE VINHO BRANCO BRASILEIROS}

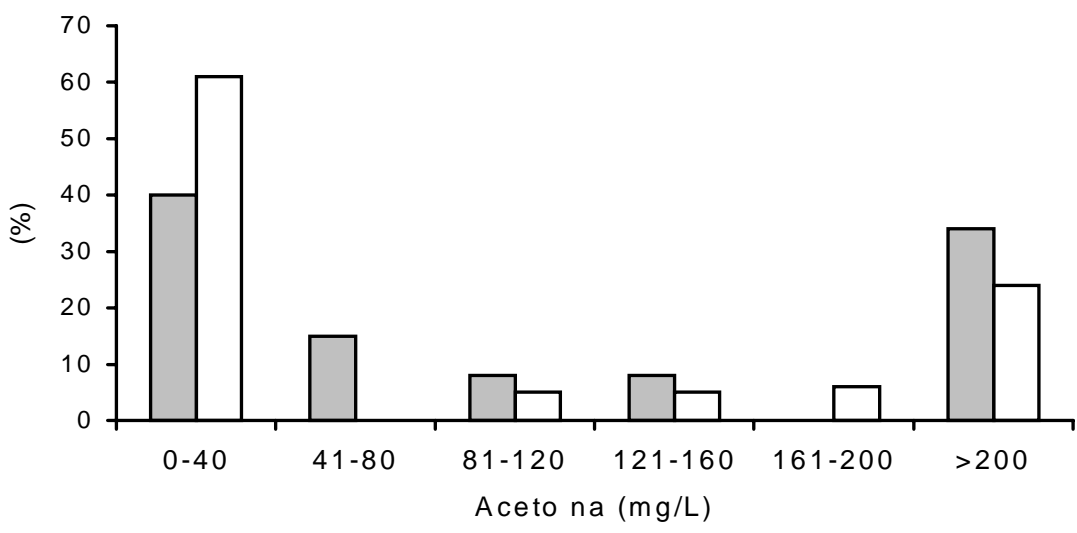

$\square$ Vinagre de vinho tinto $\square$ Vinagre de vinho branco 
Observou-se que $61,0 \%$ das amostras de vinagres de vinho branco e 39,4\% dos vinagres de vinho tinto apresentaram menos de $40 \mathrm{mg} / \mathrm{L}$ de acetoína, abaixo do limite mínimo estabelecido pela legislação espanhola (LLAGUNO e POLO, 1991). Somente $22,2 \%$ dos vinagres de vinho branco e $32,1 \%$ dos vinagres de vinho tinto continham mais de $200 \mathrm{mg} / \mathrm{L}$, mínimo de acetoína para vinagre de vinho recomendado por CURZEL (1955).

Os vinagres elaborados com álcool apresentaram apenas traços de acetoína na sua composição, proveniente do substrato fornecido para o desenvolvimento das bactérias acéticas no início do processo de acetificação (GALOPPINI e ROTINI, 1956). O vinagre de maçã, por sua vez, apresenta teor de acetoína comparável ao do vinagre de vinho. Nesse sentido, o teor de acetoína permite diferenciar o vinagre de álcool do vinagre de vinho, mas não exerce efeito discriminante para separar o vinagre de vinho do vinagre de maçã.

A diferença significativa constatada entre o teor de metanol do vinagre de vinho tinto e do vinho branco (Tabela 1) deve-se à concentração mais elevada desse álcool no vinho tinto, em razão da maceração (BERTRAND, 1975; RIZZON, 1985). Os vinhos utilizados na acetificação, geralmente elaborados com uvas do grupo das americanas, apresentam teor de metanol mais elevado (BERSELLI, 1997; GUERRA, 1998).

A distribuição percentual das amostras de vinagre analisados em diferentes níveis de metanol está indicada na Figura 2.

\section{FIGURA 2 - DISTRIBUIÇÃO DO TEOR DE METANOL EM VINAGRES DE VINHO TINTO E DE VINHO BRANCO BRASILEIROS}

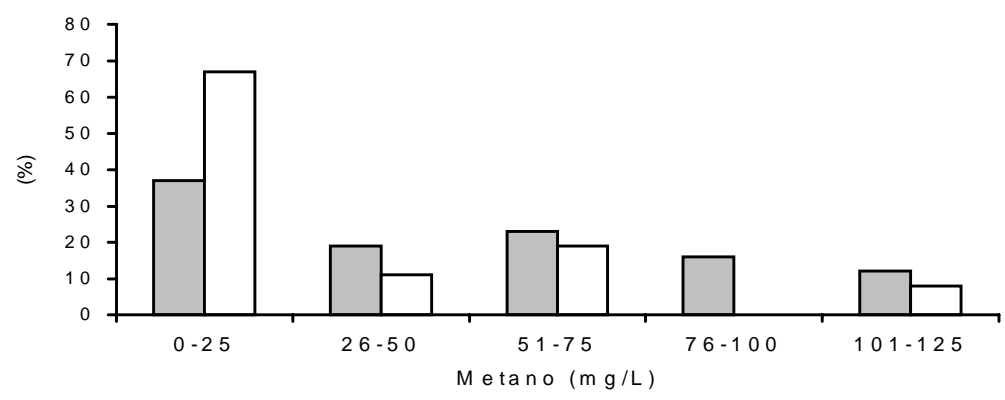

$\square$ Vinagre de vinho tinto $\square$ Vinagre de vinho branco 
Constatou-se que $66,6 \%$ dos vinagres de vinho branco e $53,6 \%$ dos vinagres de vinho tinto analisados apresentaram, respectivamente, menos de $25 \mathrm{mg} / \mathrm{L}$ e de $50 \mathrm{mg} / \mathrm{L}$ de metanol (Figura 2), portanto, abaixo dos valores médios recomendáveis para os vinagres italianos (MECCA et al., 1979). Os vinagres de vinho branco e, especialmente, os de vinho tinto com teores de metanol mais elevados foram elaborados com vinhos mais macerados e correspondem aos produtos genuínos. O vinagre elaborado a partir do álcool de cana-de-açúcar contém concentração reduzida de metanol. O vinagre de maçã, por sua vez, apresenta teor de metanol comparável ao do vinho. Assim é possível diferenciar o vinagre de álcool dos vinagres de vinho e de maçã.

Os métodos cromatográficos empregados possibilitaram a separação dos alcoóis em vinagres de vinho (tinto e branco).

\section{CONCLUSÃO}

Os resultados das análises evidenciaram que mais de $60 \%$ dos vinagres de vinho branco e $39 \%$ dos de vinho tinto apresentaram valores baixos de acetoína para vinagres de vinho (menos de $40 \mathrm{mg} / \mathrm{L}$ ). Em relação ao metanol, mais de $65 \%$ dos vinagres de vinho branco e $50 \%$ dos de vinho tinto também apresentaram valores considerados baixos para vinagres de vinho (menos de $25 \mathrm{mg} / \mathrm{L}$ e $50 \mathrm{mg} / \mathrm{L}$, respectivamente).

O estabelecimento de limite mínimo para a acetoína e o metanol do vinagre de vinho pela legislação brasileira contribuiria para a determinação da genuinidade desse produto.

A cromatografia a gás mostrou-se adequada à determinação de acetoína e metanol em vinagres, pois permite separar os componentes e apresenta elevada sensibilidade analítica.

\section{Abstract}

DETERMINATION OF ACETOIN AND METHANOL IN BRAZILIAN WINE VINEGARS The aim of this work was to characterize Brazilian wine vinegars, by determination of acetoin and methanol content. Twenty-nine samples of different commercial red wine vinegars and 18 samples of white wine vinegars were analysed. The analyses of acetoin and methanol were made by gas chromatography. High variability in the acetoin and methanol contents were observed. It was remarked that $61.0 \%$ of the white wine vinegars and $39.4 \%$ of the red wine vinegars showed less than $40 \mathrm{mg} / \mathrm{L}$ of acetoin. It was showed that $66.6 \%$ of the white wine vinegars showed less than $25 \mathrm{mg} / \mathrm{L}$ of 
methanol and $53.6 \%$ of the red wine vinegars had less than $50 \mathrm{mg} / \mathrm{L}$ of this alcohol. The setting up of limitation by Brazilian legislation for the acetoin and methanol in the vinegar components contribute to its genuinity. The gas chromatography showed to be suitable, for acetoin and methanol determination in wine vinegars.

KEY-WORDS: ENOLOGY; VINEGAR; VOLATILE COMPOUNDS; ACETOIN; METHANOL.

\section{REFERÊNCIAS}

1 BAUMES, $R$. Les constituants volatils du stade fermentaire. In: FLANZY, C. Oenologie: fondements scientifiques et technologiques. Paris: Tec \& Doc, 1998. $1284 \mathrm{p}$.

2 BERSELLI, E. Característica físico-química e sensorial do vinho Niágara comercial. Bento Gonçalves, 1997. 21 p. Monografia (Conclusão de Curso) Curso Superior de Tecnologia em Viticultura e Enologia, EAFPJK.

3 BERTRAND, A. Recherche sur l'analyse des vins par chromatographie en phase gazeuse. Talence, 1975. 291 p. Tese (Doutorado em Enologia) Université de Bordeaux II.

4 BRASIL. Ministério da Agricultura. Secretaria Nacional de Defesa Agropecuária. Secretaria de Inspeção de Produto Vegetal, Brasília, DF. Complementação de padrões de identidade e qualidade para cerveja, vinho, vinho de frutas, fermentado de cana, saquê, filtrado doce, hidromel, jeropiga, mistela, sidra, vinagre. Brasília: Imprensa Nacional, 1974. 109 p.

5 BRASIL. Secretaria de Defesa Agropecuária, Brasília, DF. Portaria ํㅜ 30 de 4 de setembro de 2000. Metodologia de análise da razão isotópica ${ }^{13} \mathrm{C} /{ }^{12} \mathrm{C}$ em produtos e subprodutos de plantas do ciclo fotossintético $\mathrm{C}^{3}$ e $\mathrm{C}^{2}$. Diário Oficial [da] República Federativa do Brasil, Brasília, n.178, seção 1, p.18-19, 14 set. 2000.

6 CORDONNIER, R. Le methanol et ses origines dans le vin. Progrès Agricole et Viticole, Montpellier, v.104, n.13-14, p.315-318, 1987.

7 CURZEL, V. La determinazione dell'acetilmetilcarbinolo negli aceti. Rivista de Viticoltura e di Enologia, Conegliano, v.8, n. 3, p.93-96, 1955.

8 FENOCCHIO, P.; MENDES, M. Teores de metanol em alguns vinhos gaúchos. Pelotas: Instituto de Pesquisa Agropecuária do Sul, 1973. 12 p. (Ministério da Agricultura, Boletim Técnico, 83).

9 FENOCCHIO, P.; MENDES, M. Teores de metanol nos sucos de uva brasileiros. Pelotas: Instituto de Pesquisa Agropecuária do Sul, 1972. 8 p. (Ministério da Agricultura. Boletim Técnico, 82).]

10 GALOPPINI, C.; ROTINI, O. T. Ulteriori indagini sulla formazione dell'acetilmetilcarbinolo nella fermentazione acetica. Annali de la Facultà di Agraria dell'Università di Pisa, Pisa, v.17, n.1, p.99-111, 1956. 
11 GNEKOW, B.; OUGH, C.S. Methanol in wines and musts: source and amounts. American Journal of Enology and Viticulture, Davis, v.27, n.1, p.1-6, 1976.

12 GUERRA, L. Características analíticas dos vinhos de São Marcos. Bento Gonçalves, 1998. 51 p. Monografia (Conclusão de Curso) - Curso Técnico em Viticultura e Enologia, EAFPJK.

13 LEE, C.Y.; ROBINSON, W.B.; VAN BUREN, J.P. et al. Methanol in wines in relation to processing and variety. American Journal of Enology and Viticulture, Davis, v.26, n.4, p.184-187, 1975.

14 LEE, C.Y.; SMITH, N.L.; NELSON, R.R. Relationship between pectin methylesterase activity and the formation of methanol in Concord grape juice and wine. Food Chemistry, New York, v.4, n.2, p.143-148, 1979.

15 LLAGUNO, C.; POLO, M.C. El vinagre de vino. Madrid: Consejo Superior de Investigación Científicas, 1991. 238 p.

16 MARCHENA, L.C. Industrias de la fermentación. Aportación al estudio de los vinagres españoles. Revista Agroquímica y Tecnología de Alimentos, Madrid, v.12, n.3, p.356-359, 1972.

17 MECCA, F.; ANDREOTTI, R.; VERONELLI, L. L'aceto. Brescia: Edizione AEB, 1979. $433 \mathrm{p}$.

18 PISANTI, A. Ricerche sul contenuto in alcool metilico nei vini Irpini. Rivista de Viticoltura e di Enologia, Conegliano, v.14, n.12, p.425-431, 1961.

19 RIZZON, L.A. Incidence de la macération sur la composition chimique des vins. Talence, 1985. 225 p. Tese (Doutorado em Enologia - Ampelologia) Université de Bordeaux II.

20 RIZZON, L.A.; MIELE, A. Características analíticas de vinagres comerciais de vinhos brasileiros. Brazilian Journal of Food Technology, Campinas, v.1, n.1 e 2, p.25-31, 1998.

21 RIZZON, L.A.; MIELE, A.; MENEGUZZO, J.; ZANUZ, M.C. Efeito de três processos de vinificação sobre a composição química e a qualidade do vinho Cabernet Franc. Pesquisa Agropecuária Brasileira, Brasília, v.34, n.7, p.1285-1293, 1999.

22 RIZZON, L.A.; ZANUZ, M.C.; MIELE, A. Efeito da fermentação maloláctica na composição do vinho tinto. Ciência Rural, Santa Maria, v.27, n.3, p.497-500, 1997.

23 SALMON, J.M. Relations levure-milieu. In: FLANZY, C. Oenologie: fondements scientifiques et technologiques. Paris: Tec \& Doc, 1998. 1284 p.

24 SHINOHARA, T.; SHIMAZU, Y.; WATANABE, M. Dosage de l'acétoïne et du lactate 
d'éthyle dans les vins par chromatographie en phase gazeuse, et étude de leur formation dans les vins. Agricultural and Biological Chemistry Journal, Tokio, v.43, n.12, p.2569-2577, 1979.

25 TAKEMOTO, S.Y. Avaliação do teor de acetoína em vinagres como forma de verificação de sua genuinidade. Florianópolis, 2000. 116 p. Dissertação (Mestrado em Engenharia de Alimentos) - Centro Tecnológico. Departamento de Engenharia Química e Engenharia de Alimentos, UFSC.

26 TRONCOSO, G.A.M.; GUSMÁN, C. M. Acetoin content of Andalusian vinegars. Sciences des Aliments, Paris, v.7, n.3, p.499-505, 1987.

27 ZMIROU-BONNAMOUR, C. Contribution à l'étude des produits secondaires de la fermentation malolactique des vins. Talence, $1984.145 \mathrm{p}$. Tese (Doutorado em Enologia - Ampelologia) - Université de Bordeaux II. 